\title{
PERTURBATION BY TRACE CLASS OPERATORS ${ }^{1}$
}

\author{
BY R. W. CAREY AND J. D. PINCUS
}

Communicated by Gian-Carlo Rota, November 27, 1973

When are two selfadjoint operators unitarily equivalent modulo the trace class? The version of this theorem in which "trace class" is replaced by "compact" is settled by the Weyl-von Neumann theorem [1]: If $A$ and $B$ are selfadjoint operators, there exists a unitary operator $U$ such that $U A U^{*}-B$ is compact if and only if $A$ and $B$ have the same essential spectrum.

The question of trace class equivalence is more delicate and requires a study of additional invariants. Thus the Kato-Rosenblum theorem states: If $A$ and $B$ are selfadjoint operators for which $A-B$ is trace class, then $A$ and $B$ have unitarily equivalent absolutely continuous parts [1].

Our purpose in the present note is to announce the following answer to the question posed above.

THEOREM. Two bounded selfadjoint operators $A$ and $B$ are unitarily equivalent modulo the trace class if and only if

(1) $A_{a c}$ is unitarily equivalent to $B_{a c}$,

(2) essential spectrum $(A)=$ essential spectrum $(B)$,

(3) there exists a decomposition of the sets $\pi_{f}(A)$ and $\pi_{f}(B)$, the isolated eigenvalues of $A$ and $B$ of finite multiplicity

$$
\pi_{f}(A)=\pi_{\approx}(A) \text { union } \pi_{w}(A), \quad \pi_{f}(B)=\pi_{\approx}(B) \text { union } \pi_{w}(B),
$$

such that

$$
\sum_{a_{n} i n \pi \approx(A)} d\left(a_{n}, \mathrm{sp}_{\mathrm{ess}}(A)\right)+\sum_{b_{n} i n \pi \approx(B)} d\left(b_{n}, \mathrm{sp}_{\mathrm{ess}}(B)\right)<\infty,
$$

$$
\sum_{a_{n} i_{n} \pi_{w}(A)} d\left(a_{n}, g\left(a_{n}\right)\right)<\infty
$$

for some one-to-one correspondence $g$ between the points of $\pi_{w}(A)$ and $\pi_{w}(B)$.

In this statement it is to be understood that the isolated eigenvalues are counted according to their multiplicity.

The significance of conditions (a) and (b) is that if two bounded selfadjoint operators $A$ and $B$ are unitarily equivalent modulo the trace class,

AMS (MOS) subject classifications (1970). Primary 47A55; 47A20.

${ }^{1}$ This work was partially supported by the National Science Foundation. 
then for any $\delta>0$ there is a decomposition such that the sum of the three terms appearing on the left-hand sides of the inequalities of (a) and (b) is majorized by

$$
\inf _{U: \text { unitary }}\left\|U A U^{*}-B\right\|_{\text {trace }}+\delta .
$$

A similar theorem remains correct for unitary operators when the distances in hypothesis (3) are reinterpreted.

The following result plays an important role in the proof.

LEMma. Let $T$ be a bounded selfadjoint operator and suppose that $T$ has purely singular spectrum. Then for any positive number $\delta$, there exists an operator $K$ in trace class, with trace norm less than $\delta$, such that $T+K$ is diagonal.

Thus it follows that any selfadjoint operator with purely singular spectrum is the sum of a diagonal operator (that is an operator with a complete set of eigenvectors) and a trace class operator of arbitrarily small trace norm.

The proof of the Lemma is based on the de la Valle Poussin [2] decomposition theorem for measures whose use was to some extent motivated by a result of Aronszajn [3] and an example in Donoghue [4].

This result is basic for the considerations of Carey-Pincus [5]. The proof of the Theorem will appear in [6].

\section{REFERENCES}

1. N. K. Ahiezer and I. M. Glazman, The theory of linear operators in Hilbert space, GITTL, Moscow, 1950; German transl., Akademie-Verlag, Berlin, 1954; English transl., Ungar, New York, 1961. MR 13, 358; 16, 596; 41 \#9015a.

2. S. Saks, Theory of the integral, reprint, Dover, New York, 1964, Chap. 4, Sect. 9. MR 29 \#4850.

3. N. Aronszajn, On a problem of Weyl in the theory of singular Sturm-Liouville equations, Amer. J. Math. 79 (1957), 597-610. MR 19, 550.

4. W. F. Donoghue, Jr., On the perturbation of spectra, Comm. Pure Appl. Math. 18 (1965), 559-579. MR 32 \#8171.

5. R. W. Carey and J. D. Pincus, Intertwining partial isometries. II (to appear).

6. - Unitary equivalence modulo the trace class for self-adjoint operators (to appear).

Department of Mathematics, University of Kentucky, Lexington, Kentucky 40506

Department of Mathematics, State University of New York, Stony Brook, NEW YORK 11790 